\section{Evaluation of patient renal function following endovascular aneurysm repair with suprarenal fixation}

\author{
YingBin Jia, ${ }^{1}$ Yun Shi, ${ }^{2}$ XiaoDong Guan, \\ Jian Li, ${ }^{1}$ BaiMeng Zhang, ${ }^{1}$ WeiGuo Fu' \\ 'Department of Vascular Surgery of the \\ Fifth Hospital Affiliated to Sun Yat-Sen \\ University, ZhuHai; ' ${ }^{2}$ Department of \\ Vascular Surgery of Zhong Shan Hospital \\ Affiliated to Fu Dan University, \\ ShangHai, China
}

Abstract

This study aimed to assess the mid-term renal function of abdominal aortic aneurysm patients following suprarenal endovascular repair. From March 2005 to December 2009, 290 abdominal aortic aneurysm patients were included in the study and grouped according to whether they had received infrarenal or suprarenal endovascular aneurysm repair. Suprarenal endovascular aneurysm repair was performed in 173 patients, with a mean age of $72( \pm 8)$ years $(85.0 \%$ male $)$. Infrarenal endovascular aneurysm repair was performed in 117 patients, with a mean age of $71( \pm 9)$ years $(90.6 \%$ male). Preoperative and one week, 1-, 3-, 6- and 12-month postoperative serum creatinine and cystatin $C$ values were recorded. Estimated glomerular filtration rate was calculated by cystatin-based formula and Cr-based Cockcroft formula. The t-test was used to determine statistical differences between or within groups.

All patients received Talent or Zenith endograft. Patients' characteristics and operative files in the two groups were well matched. Preoperative serum creatinine and cystatin $\mathrm{C}$ were $82( \pm 8) \mu \mathrm{mol} / \mathrm{L}$ and $0.89( \pm 0.11) \mathrm{mg} / \mathrm{L}$ for suprarenal endovascular aneurysm repair, respectively, and $81( \pm 11) \mu \mathrm{mol} / \mathrm{L}$ and 0.87 $( \pm 0.15) \mathrm{mg} / \mathrm{L}$, respectively, for infrarenal endovascular aneurysm repair; no differences were observed between the two groups. Compared to preoperative renal markers within each group, a deterioration in serum creatinine, cystatin $\mathrm{C}$ and estimated glomerular filtration rate values was found at one week and 12 months after surgery $(\mathrm{P}<0.05)$. A deterioration in cystatin $\mathrm{C}$ [SR:( $0.93 \pm 0.17) \mathrm{mg} / \mathrm{L}, \mathrm{IR}$ : $(0.92 \pm 0.31) \mathrm{mg} / \mathrm{L}]$ and estimated glomerular filtration rate by cystatin $\mathrm{C}$ was also found at six months after surgery $(\mathrm{P}<0.05)$. However, no differences in patient serum creatinine, cystatin $\mathrm{C}$ and estimated glomerular filtration rate values were observed between groups at each follow-up time interval. There was no greater significant difference in the association of the use of suprarenal fixation with midterm postoperative renal injury than with infrarenal fixation.

\section{Introduction}

Abdominal aortic aneurysm (AAA) is currently the most common vascular disease in the elderly in China. Renal impairment is a common risk of mortality following conventional open AAA surgery. Endovascular aneurysm repair (EVAR) has advantages over open surgery. The risk of renal hypoperfusion secondary to hemodynamic instability and cross clamping is eliminated, surgical trauma is reduced, and ischemia-reperfusion injury is attenuated. The fixation strategy in EVAR can be defined as suprarenal (SR) or infrarenal (IR) fixation according to the proximal position of the stent across the renal artery ostia. In evaluating transrenal fixation, one question is whether the bared metal stents across the renal artery ostia used in SR fixation harm renal function more than IR fixation. Although some studies showed the relative safety of this technique, ${ }^{1-5}$ most reports drew their conclusions from the study of small numbers of patients or used insensitive markers of renal function, leading to an imprecise assessment.

The purpose of this study was to evaluate the mid-term renal function of AAA patients following suprerenal EVAR compared with infrarenal, using cystatin C (Cys-C) and glomerular filtration rate (GFR) calculated by cystatin C-based equation as the sensitive markers of renal function compared with serum creatinine (SCr) and GFR calculated by the SCr-based method.

\section{Materials and Methods}

\section{Patients' demographics and risk factors}

From March 2005 to December 2009, 438 patients with infrarenal AAAs in two hospitals were entered retrospectively into a vascular registry for this study. Informed consent was obtained from all patients before surgery. Of these, 148 patients were excluded from analysis because of selective open surgery $(n=31$, $7.1 \%)$, perioperative death $(\mathrm{n}=18,4.1 \%)$, loss to follow up ( $\mathrm{n}=46,10.5 \%)$, and preoperative SCr more than 130 umol/L or preoperative Cys$\mathrm{C}$ more than $1.55 \mathrm{mg} / \mathrm{L}(\mathrm{n}=53,12.1 \%)$. The remaining 290 patients were divided into two groups according to whether they had received infrarenal or suprarenal EVAR.
Correspondence: YingBin Jia, MeiHua East Road 52, The Fifth Hospital Affiliated to Sun Yat-Sen University, ZhuHai City, GuangDong Province, China, 519000.

Tel. 86.13824110286.

E-mail: cineplasticj@yeah.net

Key words: aortic aneurysm, abdominal, renal insufficiency, vascular surgical procedures, prostheses and implants.

Received for publication: 17 June 2011. Revision received: 12 August 2011. Accepted for publication: 12 August 2011.

This work is licensed under a Creative Commons Attribution NonCommercial 3.0 License (CC BYNC 3.0).

(C) Copyright Y.B. Jia et al., 2011

Licensee PAGEPress, Italy

Surgical Techniques Development 2011; 1:e10 doi:10.4081/std.2011.e10

\section{Endovascular AAA repair}

All procedures were carried out in an operating theater equipped with a mobile C-arm (Siemens, Siremobil 2000, Erlangen, Germany) with the patient under general anesthesia. Bifurcated self-expanding endograft were implanted primarily according to availability at the time of AAA repair. Talent (Medtronic, Santa Rosa, CA, USA) were used in $92(31.7 \%)$ patients and Zenith (Cook Inc., Bloomington, IN, USA) in $198(68.3 \%)$ patients. Endograft oversize was $10-15 \%$ more than diameter of the proximal and distal necks. Contrast (Omnipaque-300) was delivered by power injection and the volume was recorded for each procedure.

\section{Postoperative follow up}

For each patient, 64-slice helical computed tomography angiography (CTA) was used to measure the length and diameter of the proximal and distal necks and to ascertain AAA geometry before surgery and one, 6 and 12 months post surgery. $\mathrm{SCr}$ and $\mathrm{Cys}-\mathrm{C}$ values were measured before surgery and again at one week, one, 3, 6 and 12 months post surgery. Estimated GFR (eGFR) were calculated by the SCr-based Cockcroft-Gault method: [ $(140$ age $) \times$ weight $(\mathrm{kg}) \times 88.4 /(72 \times \mathrm{Cr})$ for males, $(140$ - age $) \times$ weight $(\mathrm{kg}) \times 88.4 \times 0.85 /(72 \times$ $\mathrm{Cr}$ ) for females] or by cystatin-based formula: 74.835/cystatin C (mg/L) 1.333.6.

\section{Definition of renal function}

Basline renal insufficiency was defined as $\mathrm{SCr}$ more than $130 \mu \mathrm{mol} / \mathrm{L}$ or Cys-C more than $1.55 \mathrm{mg} / \mathrm{L}$. Patients with preoperative renal insufficiency were excluded from this study. 
Postoperative renal impairment was defined as an increase in $\mathrm{SCr}$ or Cys-C of more than $20 \%$.

\section{Statistical analysis}

Data of SCr, Cys-C and GFR were expressed as mean and standard deviation. T-tests were used to compare these data between preoperative and each follow-up time intervals. T-tests were also used to analyze data between groups. Non-continuous data were expressed as percentage and analyzed by $\chi^{2}$ test. Differences were considered significant if $P$ value was less than 0.05 . All statistical analyses were performed using Stata/SE 10.0 software.

\section{Results}

Infrarenal fixation was performed in 173 patients (IR group, $n=173$ ). Suprarenal fixation was performed in 117 patients (SR group, $\mathrm{n}=117$ ) because of narrow proximal landing zone $(n=65)$, severe angulation of aneurysm necks $(\mathrm{n}=32)$, or implanting a transrenal cuff to treat proximal endoleak $(n=20)$. Also, in 21 patients, only the lower renal arteries were covered by bared stent. The two side renal arteries were covered in 96 patients. Patients' characteristics were well matched between groups (Table 1). No renal ostium was blocked by covered graft.

There were no differences in $\mathrm{SCr}$, Cys-C and eGFR values between the two patient groups at each follow-up time interval. However, comparison of postoperative $\mathrm{SCr}$ with preoperative levels revealed a significant increase at one week (SR: $\mathrm{P}=0.017$, IR: $\mathrm{P}=0.010$ ) and 12 months $(\mathrm{P}<0.01)$ post surgery in $\mathrm{SR}$ and IR groups. Cys-C in the two groups post surgery, apart from at one week (SR: $\mathrm{P}<0.01$, IR: $\mathrm{P}=0.014)$ and at 12 months $(\mathrm{P}<0.01)$ post surgery, were significantly higher than preoperative values; differences in elevation were also found at six months post surgery (SR: $\mathrm{P}=0.022$, IR: $\mathrm{P}<0.01)$. eGFR caculated by cystatin $\mathrm{C}$ based equation, decreased significantly at one week and at 6 and 12 months post surgery compared to preoperative values in each group $(\mathrm{P}<0.01)$. However, eGFR calculated by SCrbased Cockcroft-Gault method only decreased at one week and at 12 months post surgery $(\mathrm{P}<0.01)$ (Tables 2 and 3).

Although a postoperative worsening of renal function was observed, values up to the definition of renal impairment only occurred in 7 (6.0\%) SR patients and 9 (5.2\%) IR patients; there was no statistical difference $(\mathrm{P}=0.775)$. These 16 patients were followed-up for more than 12 months and $\mathrm{SCr}$ and Cys-C values remained significantly elevated; however, none of these patients progressed to renal dysfunction or required dialysis.

\section{Discussion}

Suprarenal endograft fixation is increasingly common in endovascular aneurysm repair, especially in patients with suboptimal proximal aortic neck anatomy. Positioning bare metal struts across the renal ostia aims to prevent stent-graft migration, proximal endoleak, ${ }^{7}$ complications due to aortic neck angulation, ${ }^{8}$ and to increase the proportion of AAA patients with access to endovascular repair. ${ }^{9}$ However, since the bare struts across the renal ostia seem to stand in the way of renal blood flow, we hypothesize a potential additive adverse effect on renal function. The possible connection between renal function and suprarenal stent has been the subject of vigorous debate over the past dacade..$^{1,10-15}$ Renal stenosis, occlusion, an increase in SCr levels, and renal dysfunction have all been reported after SR fixation. ${ }^{16-19}$ But more studies have revealed the relative safety of SR fixation without significant renal damage. ${ }^{1-5}$

In this study, $\mathrm{SCr}$, Cys-C and eGFR values did not worsen significantly more in patients with suprarenal EVAR than those with infrarenal EVAR. A relative safe effect on renal function of a suprarenal endovascular device was confirmed. Similarly, Cotroneo et al. ${ }^{20}$ studied 60 patients with an SR stent graft and 42 patients with IR fixation and concluded that the use of endografts with suprarenal fixation did not lead to any significant increase in morphological and/or functional renal complications compared with those with infrarenal fixation. Lalka et al., ${ }^{2}$ in a prospective analysis of data from 104 patients, found that the suprarenal fixation does not cause RA stenosis, occlusion, or infarction; nor does it preclude post-EVAR renal artery intervention.

Although no statistical differences were shown between groups, a deterioration in renal function was found at one week post sur-

Table 1. Patients' characteristics and operative data.

\begin{tabular}{lccc} 
& $\begin{array}{c}\text { Suprarenal } \\
\mathrm{n}=117\end{array}$ & $\begin{array}{c}\text { Infrarenal } \\
\mathrm{n}=173\end{array}$ & $\mathrm{P}$ \\
Mean age (years) & $70.6 \pm 9$ & $71.8 \pm 8$ & 0.235 \\
Male (\%) & 90.6 & 85.0 & 0.159 \\
\hline Hypertension (\%) & 72.6 & 74.0 & 0.800 \\
CAD (\%) & 79.5 & 78.0 & 0.767 \\
\hline Diabetes (\%) & 9.4 & 6.9 & 0.446 \\
AAA size (mm) & $54.3 \pm 8.3$ & $55.6 \pm 11.5$ & 0.294 \\
\hline Contrast volume (mL) & $73 \pm 15$ & $75 \pm 21$ & 0.375
\end{tabular}

Table 2. Renal function of pre-and post-suprarenal EVAR.

$\begin{array}{lclcc} & \begin{array}{c}\mathrm{SCr} \\ (\mathrm{umol} / \mathrm{L})\end{array} & \begin{array}{c}\text { Cys-C } \\ (\mathrm{mg} / \mathrm{L})\end{array} & \begin{array}{c}\text { eGFR }(\mathrm{mL} / \mathrm{min}) \\ \text { by Cys-c }\end{array} & \text { by SCr } \\ \text { pre-EVAR } & 82 \pm 8 & 0.89 \pm 0.11 & 63.1 \pm 3.1 & 65.3 \pm 5.6 \\ \text { post-EVAR } & 98 \pm 11^{\#} & 1.01 \pm 0.15^{\#} & 55.6 \pm 4.8^{\#} & 56.8 \pm 5.2^{\#} \\ \text { lwk } & 83 \pm 12 & 0.90 \pm 0.13 & 62.4 \pm 5.1 & 64.5 \pm 6.3 \\ 1 \mathrm{mo} & 83 \pm 9 & 0.88 \pm 0.10 & 63.8 \pm 3.4 & 65.8 \pm 6.5 \\ 3 \mathrm{mo} & 84 \pm 8 & 0.93 \pm 0.17^{\#} & 58.2 \pm 3.2^{\#} & 63.7 \pm 5.9 \\ 6 \mathrm{mo} & 91 \pm 15^{\#} & 1.03 \pm 0.20^{\#} & 54.5 \pm 5.7^{\#} & 58.4 \pm 6.8^{\#} \\ 12 \mathrm{mo} & & \end{array}$

EVAR, endovascular aneurysm repair; SCr: serum creatinine (umol/); Cys-C, serum cystatin-C (mg/L); eGFR, estimated glomerular filtration rate (mL/min) calculated by cystatin-based formula. Significant difference compared with preoperative values ( $\mathrm{P}<0.05)$. No significant difference between groups $(\mathrm{P}>0.05)$.

Table 3. Renal function of pre- and post infrarenal EVAR

$\begin{array}{lcccc} & \begin{array}{c}\mathrm{SCr} \\ (\mathrm{umol} / \mathrm{L})\end{array} & \begin{array}{c}\text { Cys-C } \\ (\mathrm{mg} / \mathrm{L})\end{array} & \begin{array}{c}\mathrm{eGFR}(\mathrm{mL} / \mathrm{min}) \\ \text { by Cys-c }\end{array} & \text { by SCr } \\ \text { pre-EVAR } & 81 \pm 11 & 0.87 \pm 0.15 & 64.5 \pm 3.8 & 66.7 \pm 6.2 \\ \text { post-EVAR } & 95 \pm 13^{\#} & 0.99 \pm 0.10^{\#} & 56.7 \pm 4.9^{\#} & 57.6 \pm 7.8^{\#} \\ \text { 1wk } & 83 \pm 9 & 0.90 \pm 0.11 & 62.4 \pm 5.3 & 63.3 \pm 6.3 \\ 1 \mathrm{mo} & 82 \pm 11 & 0.87 \pm 0.09 & 64.5 \pm 5.5 & 65.3 \pm 6.6 \\ 3 \mathrm{mo} & 83 \pm 9 & 0.92 \pm 0.13^{\#} & 61.0 \pm 5.1^{\#} & 63.4 \pm 6.1 \\ 6 \mathrm{mo} & 90 \pm 12^{\#} & 1.02 \pm 0.21^{\#} & 55.0 \pm 6.2^{\#} & 57.8 \pm 6.7^{\#} \\ 12 \mathrm{mo} & & \end{array}$

EVAR, endovascular aneurysm repair; SCr: serum creatinine ( $\mu \mathrm{mol} / \mathrm{l})$; Cys-C, serum cystatin-C (mg/L); eGFR, estimated glomerular filtration rate $(\mathrm{mL} / \mathrm{min})$ calculated by cystatin-based formula. Significant difference compared with preoperative values $(\mathrm{P}<0.05)$. No significant difference between groups $(\mathrm{P}>0.05)$ 
gery. There are rational explanations for this. Firstly, controlled intraoperative hypotension when using endografts results in transient renal ischemia. Secondly, a temporary intraoperative femoral artery block causes lower limb ischemia-reperfusion injury. Also, contrast nephropathy is an acute impairment; renal function starts to deteriorate within $24 \mathrm{~h}$, peaking at about 3-5 days after contrast administration. ${ }^{21}$ We also found all markers of renal function deteriorated at 12 months post surgery. The fact that there is some decline in renal function due to increasing age may also be a reason. Alsac et al. ${ }^{22}$ found a $10 \%$ per annum deterioration in creatinine clearance in patients over 75 years of age. In addition, each patient had at least 3 contrast-enhanced CT scans during this study period. Repeated frequent contrast exposure may mimic chronic oxidative injury, resulting in a steady decline in renal function. To limit this effect, the use of magnetic resonance angiography or duplex ultrasonography should be considered as an alternative to CTA, especially in those patients at high risk of developing contrast induced nephropathy. ${ }^{23,24}$

Moreover, if using Cys-C or Cys-C-based eGFR as markers, we found a deterioration in renal function not only at one week and 12 months, but also at six months post surgery. The results confirmed that Cys-C and eGFR calculated by cystatin C-based equation could be more sensitive and could reflect the potential renal impairment earlier. Most studies assessed the effect of SR fixation on patient renal function by serial measurement of the biochemical markers SCr and Cockcroft-Gault formulated $\mathrm{CCr}$. However, $\mathrm{SCr}$ is not a sensitive indicator of renal damage and alterations in its levels may be caused by a number of factors, such as muscle mass and protein intake. Cockcroft-Gault formula underestimated patients' GFR. ${ }^{25}$ Age and body mass are important factors in estimating bias. Many reports suggest using Cys-C as markers, ${ }^{1,5,13}$ but few studies have gone any further. In this study, Cys- $\mathrm{C}$ and Cys-C-based eGFR values were used to assess renal impairment. Cys- $\mathrm{C}$ is not thought to be affected by sex, age, or weight, ${ }^{6}$ and not even thought to be influenced by infections, liver diseases, or inflammatory diseases. ${ }^{26}$ Moreover, there can be a significant increase in its plasma concentration with a reduction in minimal, subclinical mild glomerular filtration rate (GFR), allowing a more sensitive and possibly an earlier detection of renal dysfunction. ${ }^{27,28}$ in addition, Qutb et al. ${ }^{6}$ suggest that the best correlation, highest precision and least bias were seen when using a cystatin- $\mathrm{C}$ based formula.

Another advantage in this study was that 53 patients with pre-existing renal impairment were excluded. Pre-existing renal impairment was considered to have an independent effect on postoperative renal function. ${ }^{29,30}$ The purpose of this study was to assess the effects of SR fixation on renal function. So, excluding patients with pre-existing renal impairment from this study reduced influencing factors and improved the accuracy of our results.

However, there are limitations to this study. Patients were studied retrospectively and divided into non-randomized groups. As a result, selection and reporting biases are likely to have occured, even though the use of exclusion criteria should limit this effect. Meanwhile, a retrospective study can limit the amount of information to be gathered about renal artery ostial morphology and renal infarction. In addition, effects on renal function of SR fixation can not be analyzed in more detail. Also, two different endografts (Talent and Zenith) were used in this study and their use was not matched across groups. Different proximal stent struts, barbs, and an unmatched quantity of endograft all add to the complexity of analyzing results. Finally, a one year follow-up period is not long enough to be able to form any useful conclusions and a longterm study needs to be performed.

In conclusion, compared to IR fixation, the use of SR fixation was not significantly associated with medium-term postoperative renal injury. Cys-C and eGFR caculated by cystatin Cbased equation could be more sensitive and could reflect the potential renal impairment earlier. Further long-term studies are required to confirm this.

\section{References}

1. O'Donnell ME, Sun Z, Winder RJ, et al. Suprarenal fixation of endovascular aortic stent grafts: assessment of medium-term to long-term renal function by analysis of juxtarenal stent morphology. J Vasc Surg 2007;45:694-700.

2. Lalka S, Johnson M, Namyslowski J, et al. Renal interventions after abdominal aortic aneurysm repair using an aortic endograft with suprarenal fixation. Am J Surg 2006; 192:577-82.

3. England A, Butterfield JS, Ashleigh RJ. Incidence and effect of bare suprarenal stent struts crossing renal ostia following EVAR. Eur J Vasc Endovasc Surg 2006;32:523-8.

4. Parmer SS, Carpenter JP; Endologix Investigators. Endovascular aneurysm repair with suprarenal vs infrarenal fixation: a study of renal effects. J Vasc Surg 2006;43:19-25.

5. Davey P, Rose JD, Parkinson T, et al. The mid-term effect of bare metal suprarenal fixation on renal function following endovascular abdominal aortic aneurysm repair. Eur J Vasc Endovasc Surg 2006;32:516-22. Epub 2006 Jun 15.

6. Qutb A, Syed G, Tamim HM, et al. Cystatin C-based formula is superior to MDRD, Cockcroft-Gault and Nankivell formulae in estimating the glomerular filtration rate in renal allografts. Exp Clin Transplant 2009;7:197-202.

7. Sternbergh WC 3rd, Money SR, Greenberg RK, et al.; Zenith Investigators. Influence of endograft oversizing on device migration, endoleak, aneurysm shrinkage, and aortic neck dilation: results from the Zenith Multicenter Trial. J Vasc Surg 2004;39:20-6.

8. Robbins M, Kritpracha B, Beebe HG, et al. Suprarenal endograft fixation avoids adverse outcomes associated with aortic neck angulation. Ann Vasc Surg 2005;19: 172-7.

9. Arko FR, Filis KA, Seidel SA, et al. How many patients with infrarenal aneurysms are candidates for endovascular repair? The Northern California experience. J Endovasc Ther 2004;11:33-40.

10. Zayed HA, Bell RE, Clough RE, et al. Results of endovascular repair of abdominal aortic aneurysms with an unfavorable proximal neck using large stent-grafts. Cardiovasc Intervent Radiol 2009;32:11614.

11. Sun Z, Chaichana T. Investigation of the hemodynamic effect of stent wires on renal arteries in patients with abdominal aortic aneurysms treated with suprarenal stent-grafts. Cardiovasc Intervent Radiol 2009;32:647-57. Epub 2009 Mar 17.

12. García JM, Monzón EO, Martínez AP, et al. Comparative analysis of renal function after treatment of infrarenal abdominal aortic aneurysms with a suprarenal fixation device as opposed to open surgery. Ann Vasc Surg 2008;22:513-9.

13. Sun Z, O'Donnell ME, Winder RJ, et al. Effect of suprarenal fixation of aortic stent-grafts on the renal artery ostia: assessment of morphological changes by virtual intravascular endoscopy. $\mathrm{J}$ Endovasc Ther 2007;14:650-60.

14. Mills JL Sr, Duong ST, Leon LR Jr, et al. Comparison of the effects of open and endovascular aortic aneurysm repair on long-term renal function using chronic kidney disease staging based on glomerular filtration rate. J Vasc Surg 2008;47: 1141-9.

15. Greenberg RK, Chuter TA, LawrenceBrown M, et al.; Zenith Investigators. Analysis of renal function after aneurysm repair with a device using suprarenal fixation (Zenith AAA Endovascular Graft) in contrast to open surgical repair. J Vasc Surg 2004;39:1219-28. Erratum in: J Vasc Surg 2004;40:23. 
16. Alric P, Hinchliffe RJ, Picot MC, et al. Longterm renal function following endovascular aneurysm repair with infrarenal and suprarenal aortic stent-grafts. J Endovasc Ther 2003;10:397-405.

17. Subedi SK, Lee AM, Landis GS. Suprarenal fixation barbs can induce renal artery occlusion in endovascular aortic aneurysm repair. Ann Vasc Surg 2010;24:113.e7113.e10.

18. Böckler D, Krauss M, Mansmann U, et al. Incidence of renal infarctions after endovascular AAA repair: relationship to infrarenal versus suprarenal fixation. J Endovasc Ther 2003;10:1054-60.

19. Krämer SC, Seifarth H, Pamler R, et al. Renal infarction following endovascular aortic aneurysm repair: incidence and clinical consequences. J Endovasc Ther 2002;9:98-102.

20. Cotroneo AR, Iezzi R, Giancristofaro D, et al. Endovascular abdominal aortic aneurysm repair and renal complications: a comparison between suprarenal and infrarenal fixation of stent grafts. Radiol Med 2007;112:252-63.

21. Lindholt JS. Radiocontrast induced nephropathy. Eur J Vasc Endovasc Surg 2005:25:296-304.

22. Alsac JM, Zarins CK, Heikkinen MA, et al. The impact of aortic endografts on renal function. J Vasc Surg 2005;41:926-30.

23. Lau LL, Hakaim AG, Oldenburg WA, et al. Effect of suprarenal versus infrarenal aortic endograft fixation on renal function and renal artery patency: a comparative study with intermediate follow-up. J Vasc Surg 2003;37:1162-8.

24. Surowiec SM, Davies MG, Fegley AJ, et al. Relationship of proximal fixation to postoperative renal dysfunction in patients with normal serum creatinine concentration. J Vasc Surg 2004;39:804-10.

25. Pedone C, Corsonello A, Incalzi RA. Estimating renal function in older people: a comparison of three formulas. Age
Ageing 2006;35:121-6.

26. Villa $\mathrm{P}$, Jiménez $\mathrm{M}$, Soriano MC, et al. Serum cystatin $\mathrm{C}$ concentration as a marker of acute renal dysfunction in critically ill patients. Crit Care 2005;9:R139-43.

27. Coll E, Botey A, Alvarez L, et al. Serum cystatin $\mathrm{C}$ as a new marker for noninvasive estimation of glomerular filtration rate and as a marker for early renal impairment. Am J Kidney Dis 2000;36:29-34.

28. Reed CH. Diagnostic applications of cystatin C. Brit J Biomed Sci 2000;57:323-9.

29. Black SA, Brooks MJ, Naidoo MN, et al. Assessing the impact of renal impairment on outcome after arterial intervention: a prospective review of 1,559 patients. Eur J Vasc Endovasc Surg 2006;32:300-4.

30. van Eps RG, Leurs LJ, Hobo R, et al. Impact of renal dysfunction on operative mortality following endovascular abdominal aortic aneurysm surgery. Br J Surg 2007;94:1748. 